\title{
АНАЛИЗ УСЛОВИЙ РЕШЕНИЯ ЗАДАЧИ РАСПРЕДЕЛЕНИЯ РЕСУРСОВ С ОГРАНИЧЕНИЯМИ ФАКТОРНОГО ПРОСТРАНСТВА
}

\author{
О. А. Коновалов, Е. В. Коновальчук, Р. А. Прохорский, Д. С. Винокуров \\ ВУНЦ ВВС «Военно-воздушная академия им. проф. Н. Е. Жуковского и Ю. А. Гагарина» (г. Воронеж)
}

\section{Поступила в редакцию 28.06.2018 г.}

\begin{abstract}
Аннотация. Рассматривается задача распределения ресурсов по командам в ходе реализации мультипроекта. Приведена постановка и формулировка задачи с ограничениями на факторное пространство и область изменения целевой функции в терминах математического программирования. Показано, что решение в области заданных ограничений возможно получить при использовании эвристических методов и приемов.

Ключевые слова: распределение ресурсов, сетевая модель, математическая модель.

Annotation. The problem of resources distribution into teams is considered during the implementation of the so-called "multiproject". The blow of the statement and formulation of the task with the restrictions for the factor space and the area of the efficiency function change is given in the terms of the mathematical programming. It is clearly shown that it is possible to find the solution in the field of the input restraints with the use of the heuristic methods and receptions. Keywords: distribution of resources, network model, mathematical model.
\end{abstract}

\section{ВВЕДЕНИЕ}

В настоящее время определенный интерес представляют задачи оптимального распределения потоков ресурсов на сетях. В общем случае выбор состава и распределение ресурсов, реализующих набор алгоритмов, являются одной из наиболее сложных задач на этапах планирования и проектирования сетей [1]. При этом процессы планирования операций (работ) и распределения потоков ресурсов (складируемых или нескладируемых) представляют два взаимосвязанных аспекта этой проблемы.

Задачи со строгими ограничениями на складируемые ресурсы сводятся к задачам математического программирования (МП) с выпуклыми ограничениями и целевой функцией (ЦФ), структура которой зависит от стоимости работ и их продолжительностей. При этом классические методы МП в ряде случаев могут отрицательно сказываться на достоверности результатов из-за сильных ограничений на факторное пространство и область

(c) Коновалов О. А., Коновальчук Е. В., Прохорский Р. А., Винокуров Д. С., 2018 изменения ЦФ. Сведение к задаче МП с ограничениями на нескладируемые ресурсы позволит получить несвязанные или многосвязные, т.е. невыпуклые ограничения.

Как правило, простой проект, для которого составляется сетевая модель (СМ), представляет собой связный сетевой граф (комплекс операций) с одним целевым событием. При этом технологическая зависимость между операциями задается в виде детерминированной сети с первоначальным планом распределения ресурсов. Однако, на практике при выполнении сложных проектов (мультипроектов) СМ могут иметь несколько целевых событий (выходов) [2], например, при решении задач перебазирования войск и развертывания техники, оборонительных и наступательных операций и т. д.

В отличие от подходов к решению подобной задачи, представленных в [3, 4], в статье предлагается рассмотреть случай, когда для выполнения проекта все исполнители подразделяются на различные команды. Задача заключается в минимизации времени проекта при введении соответствующих реальных ограничений на ресурсы и область изменения ЦФ. 
Анализ условий решения задачи распределения ресурсов с ограничениями факторного пространства

\section{ПОСТАНОВКА ЗАДАЧИ}

Рассмотрим проект $A$, состоящий из связных СМ с общими ресурсами, но с различными директивными сроками для отдельных СМ или отдельных целевых событий. Технологическая зависимость между СМ задается в виде детерминированной сети с первоначальным планом распределения ресурсов. Множество всех целевых событий проекта обозначим $E^{f}$, а директивный срок свершения события $\mu \in E^{f}-$ через $T_{\mu}^{\partial и р}$.

Пусть на реализацию проекта выделено ограниченное количество ресурсов $S$. Примем, что все исполнители сгруппированы в $g$ подразделений $N_{w}(1 \leq w \leq g)$ так, что в каждом подразделении $N_{w}$ все исполнители одинаковой квалификации и каждая операция СМ выполняется лишь в одном подразделении. При этом фиктивные операции, не требующие ни времени, ни исполнителей, и операции, обозначающие процессы, требующие только времени без исполнителей формально выполняются в подразделении без исполнителей, $N_{0}$.

Выбрав единицу времени $k$ (час, день, месяц) и длину $K$ промежутка времени, в котором будет осуществляться планирование, для каждого подразделения $N_{w}(1 \leq w \leq g)$ и каждой $k$-й единицы времени зададим число $x_{w}^{k} \geq 0$ исполнителей, предназначенных для выполнения планируемого проекта. Вся совокупность СМ проекта $A$ задается списком операций $L$, который рассматривается как список операций одной (не обязательно связной) СМ.

Каждая $l$-я операция характеризуется $i$-м предшествующим и $j$-м последующим событием, $l_{i j} \in L(i$ и $j$ вершины дуги $(i, j)$ соответственно); номером подразделения $w$, в котором выполняется операция; допустимой продолжительностью $t_{i j}^{\partial o n}\left(t_{i j}^{\partial \circ n} \geq 0\right)$, планируемым $N_{i j}$, допустимым $N_{i j}^{\text {доn }}$, минимальным $N_{i j}^{\min }$ и максимальным $N_{i j}^{\max }$ количеством исполнителей $\left(0 \leq N_{i j} \leq N_{i j}^{i o n} \leq N_{i j}^{\max }\right)$. На множестве всех операций введем частичное упорядочение: $(i, j)<(\bar{i}, \bar{j})$, если существует путь из $j$ в $\bar{i}$ (в частности при $\bar{i}=j$ ).
Для каждой $l_{i j}$-й операции, требуется определить следующие параметры: момент начала ее выполнения, $t_{i j}^{0}\left(t_{i j}^{0} \geq 0\right)$; планируемую продолжительность, $t_{i j}\left(t_{i j} \geq 0\right)$; планируемое количество исполнителей, $N_{i j}\left(N_{i j}^{\min } \leq N_{i j} \leq N_{i j}^{\max }\right)$.

Расчет параметров СМ (ранних и поздних сроков) выполняется по стандартным формулам [5]. Для многоцелевой СМ определим величину длительности операций $y_{i}$ для каждого события $i$ (с учетом текущего события $z$ ):

$$
y_{i}= \begin{cases}\max _{\mu \in E^{f}} T_{\mu}^{\text {дир }}-T_{i}^{\text {дир },} & \text { если } i \in E^{f} ; \\ \max _{z \gg i}\left[y_{z}+t_{i z}^{\text {доn }}\right], & \text { если } i \notin E^{f} .\end{cases}
$$

Для любого события $i$ наиболее позднее допустимое время его свершения (относительно планируемого) определятся в соответствии с выражением:

$$
t_{i}^{\text {nos }}=\max _{\mu \in E^{f}} T_{\mu}^{\text {дup }}-\tau_{i} .
$$

Очевидно, что из $i \ll j$ следует $y_{i} \geq y_{j}$ и соответственно $t_{i}^{\text {поз }} \leq t_{j}^{\text {поз }}$.

Положим, что $N_{i j} \cdot t_{i j}=N_{i j}^{\text {jon }} \cdot t_{i j}^{\text {дon }}=Q_{i j}$, где $Q_{i j}$ - трудоемкость операции, которая является постоянной величиной для каждой операции. Тогда $t_{i j}=\left[Q_{i j} / N_{i j}+0,5\right]$ и $N_{i j}=\left[Q_{i j} / t_{i j}+0,5\right]$ с точностью до ближайшего целого числа.

Для решения рассматриваемой задачи примем следующие ограничения:

$1^{*}$. Выполнение каждой $l_{i j}$-й операции не прерывается до ее окончания, т. е. происходит в промежутке времени $\left(\tau_{i j}, \tau_{i j}+t_{i j}\right]$.

$2^{*}$. Число исполнителей $N_{i j}$, приступивших к выполнению $l_{i j}$-й операции остается неизменным до ее окончания.

$3^{*}$. Отклонение величин $N_{i j}$ от $N_{i j}^{\text {доn }}$ и $t_{i j}$ от $t_{i j}^{\text {до }}$ допускается в случае, если невозможно соблюсти заданные директивные сроки свершения событий.

Искомые величины должны удовлетворять условиям СМ:

$$
\tau_{i j} \geq t_{i}^{\text {paH }}, t_{i}^{\text {paH }}=\max _{z<i}\left\{\tau_{z i}+t_{z i}\right\}
$$

и ограничениям по ресурсам:

$$
\sum_{l_{i j} \in L_{w}} N_{i j}^{k} \leq x_{w}^{k} ; \quad 1 \leq w \leq g ; \quad 1 \leq k \leq K
$$

где $t_{i}^{\text {ран }}$ - наиболее раннее возможное время свершения события $i$; 
$L_{w}$ - множество всех операций сетевого графика, выполняемых в $N_{w}$-м подразделении исполнителей;

$N_{i j}^{k}$ - число исполнителей, занятых выполнением $l_{i j}$-й операции на $k$-й единице времени, которое определяется из условия:

$$
N_{i j}^{k}=\left\{\begin{array}{l}
N_{i j} \text { при } k \in\left(\tau_{i j}, \tau_{i j}+t_{i j}\right] ; \\
0 \quad \text { при } k \notin\left(\tau_{i j}, \tau_{i j}+t_{i j}\right] .
\end{array}\right.
$$

При этом функционал (3) должен достигать минимума:

$$
F=\max _{\mu \in E^{f}}\left\{b_{\mu}\left[t_{\mu}^{\partial o n}-T_{\mu}^{\partial u p}\right]\right\},
$$

где $b_{\mu} \geq 0$ - заданные числа (для каждого $\left.\mu \in E^{f}\right)$;

$t_{\mu}^{\text {доn }}$ - реальное наиболее позднее допустимое время свершения события $\mu$.

Для любого события СМ $t_{\mu}^{\text {доn }}$ определяется из выражения:

$$
t_{\mu}^{\text {доn }}=\left\{\begin{array}{l}
\max _{z<\mu}\left\{t_{z \mu}+\tau_{z \mu}\right\}, \text { если } \mu \in E^{f}, \\
\min _{z>>\mu} t_{\mu z}, \text { если } \mu \notin E^{f} .
\end{array}\right.
$$

Физический смысл функционала (3) заключается в том, что каждому целевому событию $\mu$ приписывается определенный вес $b_{\mu}$, и берется максимум взвешенных отклонений планируемых сроков свершения целевых событий от директивных сроков. Этот максимум необходимо минимизировать путем выбора величин $t_{i j}^{0}$ и $N_{i j}$ при выполнении ограничений (1) и (2). Рассмотренную выше задачу для краткости будем называть эту задачу задачей $\Omega$.

Необходимо отметить, что важный частный случай задачи $\Omega$ (задача $\Sigma$ ) получим при $N_{i j} \equiv N_{i j}^{\partial o n}$, т. е. $N_{i j}^{\min }=N_{i j}^{\max }=N_{i j}^{\partial o n}$ и $t_{i j} \equiv t_{i j}^{\partial o n}$ для всех операций СМ. На практике такой случай характерен при планировании операций с установленными временными нормативами трудоемкости операций (например, при перебазировании воинских частей). Так как величины $N_{i j}$ фиксированы, то минимизация функционала $F$ происходит лишь за счет выбора значений $\tau_{i j}$.

\section{ФОРМУЛИРОВКА ЗАДАЧИ В ТЕРМИНАХ МП}

Пусть $\rho$ - число операций в СМ. Для каждой из $\rho$ операций $l_{i j} \mathrm{CM}$ и каждой из $K$ единиц времени $k$ необходимо построить функцию двух аргументов, определенную при $N_{i j} \in\left[N_{i j}^{\min }, N_{i j}^{\max }\right]:$

$$
f^{k}\left(\tau_{i j}, N_{i j}\right)=\left\{\begin{array}{l}
0, \text { при } t_{i j}^{0}<k-t_{i j} ; \\
N_{i j}, \text { при } k-t_{i j} \leq \tau_{i j} \leq k ; \\
0, \text { при } t_{i j}^{0} \geq k .
\end{array}\right.
$$

В случае $N_{i j} \equiv N_{i j}^{\text {доn }}$ функция (4) зависит только от одного аргумента. Обозначим его $f^{k}\left(\tau_{i j}\right)$. Аргументы функций (4), так же как их значения и параметр $k$, являются целочисленными. Для простоты будем считать аргументы $t_{i j}^{0}$ непрерывными. Так как в силу определения все $t_{i}^{\text {ран }}$ неотрицательны, то из (1) следует неотрицательность всех $\tau_{i j}$.

При этом значением функции $f^{k}\left(\tau_{i j}, N_{i j}\right)$ является число исполнителей, занятых на $k$-й неделе при выполнении операции $l_{i j}$, если она начата в момент $t_{i j}^{0}$ силами $N_{i j}$ исполнителей и выполняется с соблюдением условий $1^{*}$ и $2^{*}$.

Далее перепишем ограничения (2) и неравенства (1) в следующем виде:

$$
\begin{gathered}
\sum_{l_{i j} \in L_{w}} f^{k}\left(\tau_{i j}, N_{i j}\right) \leq x_{w}^{k} ; \\
1 \leq w \leq g ; \quad 1 \leq k \leq K, \\
\tau_{i j} \geq \max _{z<i}\left\{\tau_{z \mu}+t_{z \mu}\right\}, \quad l_{i j} \in L .
\end{gathered}
$$

Ограничив время выполнения проекта $A$ промежутком $(0, K]$, предположим, что для каждой $l_{i j}$-й операции выполняется неравенство:

$$
t_{i j}^{0} \leq K-\left[y_{j}+t_{i j}\right],
$$

где $y_{j}=\max _{z>>j}\left\{y_{z}+\tau_{j z}\right\}$.

Также следует отметить, что и величины $N_{i j}$ подчинены ограничениям [3]:

$$
N_{i j}^{\min } \leq N_{i j} \leq N_{i j}^{\max } .
$$

Если несколько упростить поставленную задачу $\Omega$, сняв трудноформализуемое условие $3^{*}$, то полученная задача $\Omega^{*}$ будет эквивалентна следующей задаче МП. 
В $2 r$-мерном пространстве точек $\left(\left\{t_{i j}^{0}\right\}\right.$, $\left.\left\{N_{i j}\right\}\right)$ найти целочисленную точку, доставляющую минимум функционала (3) при выполнении условий (5)-(8).

Для решения задачи $\Omega^{*}$ проанализируем ее частный случай (задачу $\Sigma$ ).

В $r$-мерном пространстве $R$ точек $D=\left\{\left\{t_{i j}^{0}\right\}\right\}$ найти целочисленную точку $D^{0}=\left\{t_{i j}^{0}\right\}$, доставляющую минимум функционала

$$
F(D)=\max _{\mu \in E^{f}}\left\{b_{\mu}\left[\max _{z<\mu}\left(t_{z, \mu}^{0}+t_{z, \mu}-T_{\mu}^{\text {¿up }}\right)\right]\right\},
$$

при ограничениях $\left(5^{*}\right)-\left(7^{\star}\right)$ :

$$
\begin{gathered}
\sum_{l_{i j} \in L_{w}} f^{k}\left(t_{i j}^{0}\right) \leq x_{w}^{k},(1 \leq w \leq g ; 1 \leq k \leq K), \\
t_{i j}^{0} \geq \max _{z<<i}\left\{t_{z i}^{0}+t_{z i}\right\}, l_{i j} \in L \\
t_{i j}^{0} \leq K-\left[y_{j}+t_{i j}\right], \quad \forall i, j, l_{(i, j)}
\end{gathered}
$$

где $b_{\mu}, T_{\mu}^{\text {дир }}, t_{z \mu}, t_{z i}, t_{i j}, x_{w}^{k}, K, y_{j}$-постоянные числа.

Функционал (9) является кусочно-линейным, а система из кусочно-линейных неравенств (6) и $r$-линейных неравенств (7) определяет ограниченную выпуклую область (многогранник) в пространстве $R$.

Таким образом, задача $\Sigma$ без ограничений по ресурсам (задача $\Upsilon$ ) является задача выпуклого кусочно-линейного программирования, точное решение которой можно найти регулярными методами [6].

\section{АНАЛИЗ ХАРАКТЕРА ОГРАНИЧЕНИЙ В ЗАДАЧАХ $\Omega, \Sigma$ И $\Upsilon$}

С помощью любого алгоритма для нахождения критического пути [7] найдем наиболее раннее возможное время $t_{\mu}^{\prime \text { ран }}$ свершения каждого события $\mu$ :

$$
t_{\mu}^{\prime \text { ран }}=\max _{z<\mu}\left\{t_{z}^{t^{\text {par }}}+t_{z \mu}\right\} .
$$

При этом если $\max t_{\mu}^{\prime \text { ран }} \leq K$, то положим

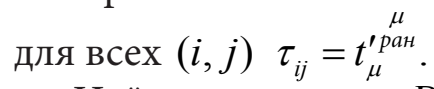

Найденная точка $D^{\prime}=\left\{\tau_{i j}=t_{i}^{\prime \text { ран }}\right\}$ доставляет минимум функционала (9) при ограничениях (6), (7). Если же $\max _{\mu} t_{\mu}^{\prime \text { ран }}>K$, то задача $\Upsilon$ не имеет решения.
Для определения всего множества решения задачи $\Upsilon$, вычислим для каждого события $\mu$ величину

$$
t_{\mu}^{\text {ран }}=\left\{\begin{array}{l}
\min \left\{T_{\mu}^{\text {дир }}+\frac{F\left(D^{\prime}\right)}{b_{\mu}}, K\right\}, \text { если } \mu \in E^{f} ; \\
\min _{z>>\mu}\left\{t_{\mu}^{\prime \prime \text { ран }}-t_{\mu z} \tau(e, z)\right\}, \text { если } \mu \notin E^{f} .
\end{array}\right.
$$

Точка $D^{\prime \prime}=\left\{\tau_{i j}=t_{\mu}^{\prime \text { ран }}\right\}$ также является решением задачи $\Upsilon$, а все множество ее решений содержится во множестве целочисленных точек параллелепипеда $D^{\prime} \leq D \leq D^{\prime \prime}$ (т. е. точек $D$, у которых для всех $(i, j)$ : $\left.t_{i}^{\prime \text { ран }} \leq \tau_{i j} \leq t_{i}^{\prime \prime \text { ран }}\right)$.

Определим характер ограничений $\left(5^{\star}\right)$ в рассматриваемой задаче $\Sigma$. Отметим, что каждая функция $f^{k}\left(\tau_{i j}\right)$ имеет П-образный график (рис. 1).

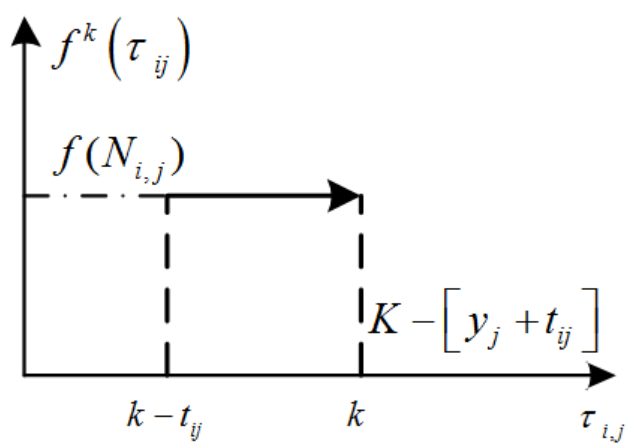

Рис. 1. График функции $f^{k}\left(\tau_{i, j}\right)$

Для случая, когда $L_{w}$ состоит из двух операций $(i, j)$ и $(\bar{i}, \bar{j})$ неравенства $\left(5^{\star}\right)$ примут вид:

$$
f^{k}\left(\tau_{i, j}\right)+f^{k}\left(\tau_{\bar{i}, \bar{j}}\right) \leq x_{w}^{k} .
$$

Из рис. 1 видно, что график левой части (10) представляет ступенчатую поверхность, представленную на рис. 2 для случая $N_{\bar{i}, \bar{j}}>N_{i, j}$.

Графиком правой части является плоскость, перпендикулярная оси $\sum f^{k}$ и проходящая на расстоянии $x_{w}^{k}$ от начала координат.

На рис. 2 представлен вариант для соотношения $f\left(N_{\bar{i}, \bar{j}}\right)>f\left(N_{i, j}\right)$. При этом три точки $\min \left\{f\left(N_{i, j}\right), f\left(N_{\bar{i}, \bar{j}}\right)\right\}, \quad \max \left\{f\left(N_{i, j}\right), f\left(N_{\bar{i}, \bar{j}}\right)\right\}$ и $f\left(N_{i, j}\right)+f\left(N_{\bar{i}, \bar{j}}\right)$ делят положительную полуось $\sum^{k} f^{k}$ на четыре промежутка.

В зависимости от того, в каком из промежутков находится величина $x_{w}^{k}$, точки удовлетворяющие неравенству (10), образуют на 


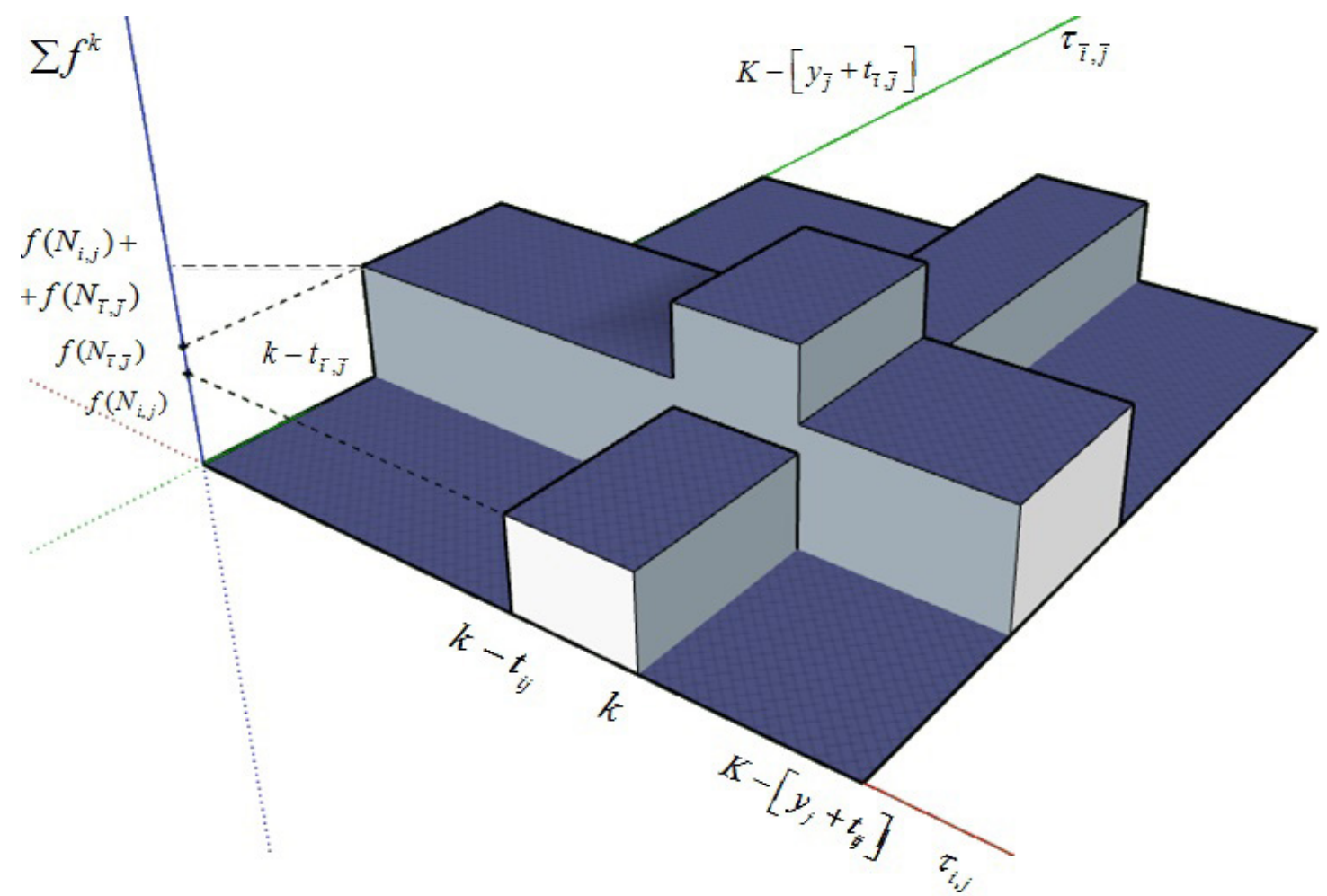

Рис. 2. График функщии $f^{k}\left(\tau_{i, j}\right)+f^{k}\left(\tau_{\bar{i}, \bar{j}}\right)$

плоскости $\left(\tau_{i, j}, \tau_{\bar{i}, \bar{j}}\right)$ одно из четырех множеств, представленных на рис. 3.

Для каждого значения $k(1 \leq k \leq K)$ неравенство будет ограничено соответствующей областью. А так как все неравенства должны выполняться одновременно, то для получения допустимой области следует взять пересечение всех полученных множеств. При этом в общем случае это будет несвязная область с одно- и многосвязными компонентами с кусочно-линейными границами.

Если $L_{w}$ состоит более чем из двух операций, то область решений неравенства (10) будет иметь тот же характер, что и $\left(5^{\star}\right)$, но большую размерность. Теперь на этой области строится цилиндрическое множество в пространстве $R$. Затем проводим аналогичное построение для другогош и т. д. Пересечение цилиндрических множеств и образует допустимую область $\left(5^{*}\right)$. Отметим, что эта область может быть выпуклой тогда и только тогда, когда все цилиндрические множества выпуклы [6]. Следовательно, все ограничения по ресурсам несущественны. Значит задача $\Sigma$ - это целочисленная задача невыпуклого кусочно-линейного программирования.
Рассмотрим теперь более подробно задачу $\Omega^{*}$. Анализ ее математической модели показывает, что для каждой операции $(i, j)$ выбор числа исполнителей $N_{i j}$ является основным, а продолжительность $t_{i j}$ является функцией от $N_{i j}$. При этом ограничения (6), (7) и функционал (9) показывают, что такая формализация приводит к появлению членов, гиперболических относительно $N_{i j}$, в неравенствах, линейных и кусочно-линейных относительно $t_{i j}$, а также в функционале, кусочно-линейном относительно $t_{i j}$. Поэтому для упрощения математической модели рассмотрим $t_{i j}$ в качестве аргументов, считая $N_{i j}$ их функциями:

$$
\begin{gathered}
N_{i j}=\left[Q_{i j} / t_{i j}+0,5\right] ; \quad t_{i j}^{\min }=\left[Q_{i j} / N_{i j}^{\max }+0,5\right] ; \\
t_{i j}^{\max }=\left[Q_{i j} / N_{i j}^{\min }+0,5\right] .
\end{gathered}
$$

Функцию (4) будем считать функцией от переменных $\tau_{i j}$ и $t_{i j}$, т. е. $f^{k}\left(\tau_{i j}, t_{i j}\right)$. Так как $f^{k}\left(\tau_{i j}, t_{i j}\right)$ - кусочно-постоянная функция, то ограничения (5) и (8) примут вид:

$$
\begin{gathered}
\sum_{l_{i j} \in L_{w}} f^{k}\left(\tau_{i j}, t_{i j}\right) \leq x_{w}^{k}, \\
(1 \leq w \leq g ; 1 \leq k \leq K),
\end{gathered}
$$


Анализ условий решения задачи распределения ресурсов с ограничениями факторного пространства
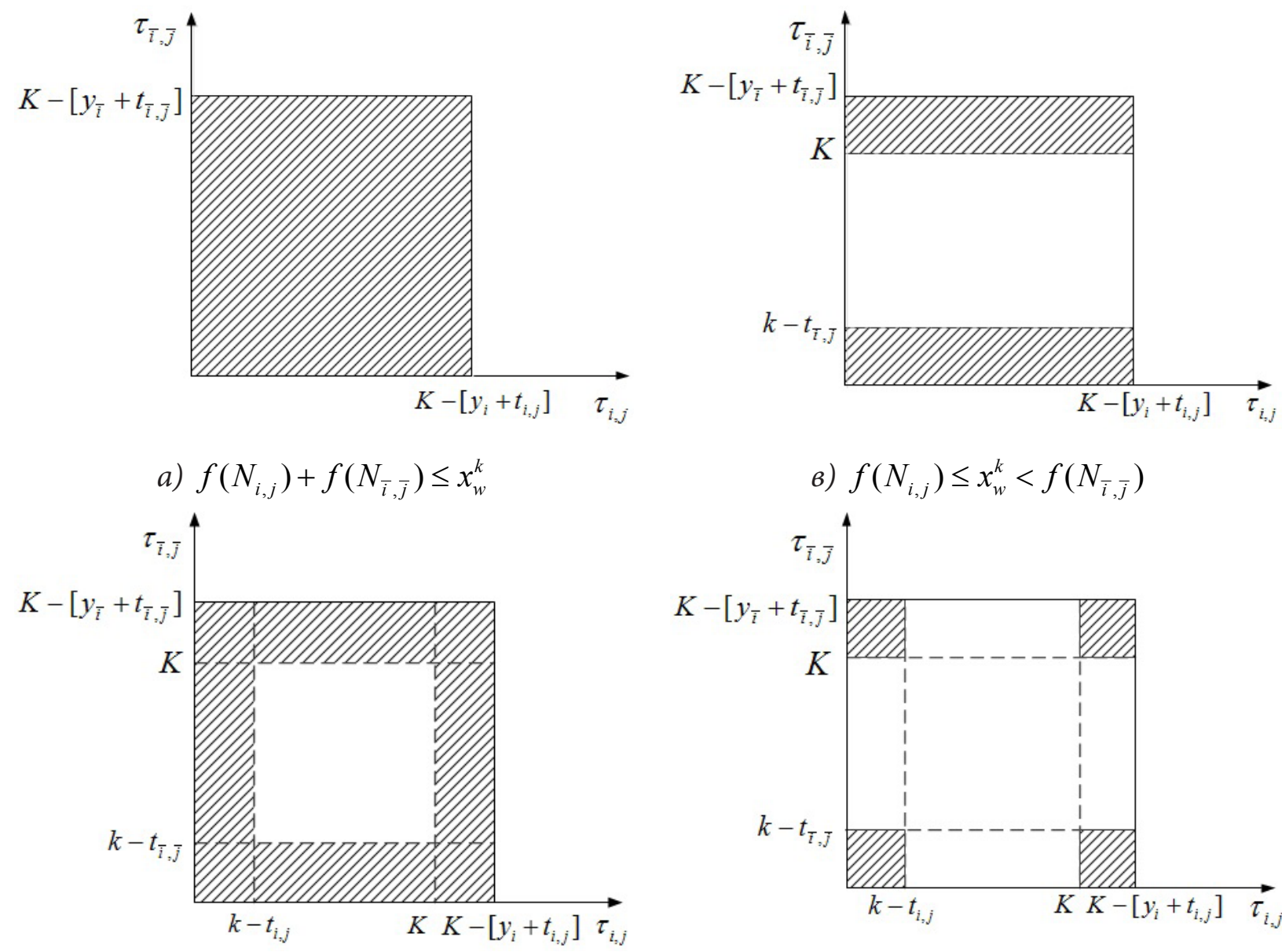

б) $\max \left\{f\left(N_{i, j}\right), f\left(N_{\bar{i}, \bar{j}}\right)\right\} \leq x_{w}^{k}<f\left(N_{i, j}\right)+f\left(N_{\bar{i}, \bar{j}}\right)$

2) $\min \left\{f\left(N_{i, j}\right), f\left(N_{\bar{i}, \bar{j}}\right)\right\}>x_{w}^{k}$

Рис. 3. Множества плоскости $\left(\tau_{i, j}, \tau_{\bar{i}, \bar{j}}\right)$

$$
t_{i j}^{\min } \leq t_{i j} \leq t_{i j}^{\max } .
$$

В новых переменных переформулируем задачу $\Omega^{*}$. В $2 r$-мерном пространстве $\tilde{R}$ точек $\tilde{D}=\left(\tau_{i j}, t_{i j}\right)$ найти целочисленную точку, доставляющую минимум функционала (9) при выполнении ограничений $\left(5^{\star *}\right),(6),(7)$ и (11).

Так как функционал (9) выпуклый кусочно-линейный, а система неравенств (11) определяет в пространстве $\tilde{R}$ прямоугольный параллелепипед (т. е. выпуклый многогранник), то и система неравенств (6) и (7) также определяет в пространстве $\tilde{R}$ выпуклый многогранник.

Совокупность неравенств (6), (7) и (11) определяет в пространстве $\tilde{R}$ выпуклый многогранник, являющийся пересечением двух указанных многогранников. Таким образом, задача минимизации времени мультипроекта при неограниченных ресурсах, одним из решений которой является точка $D^{\prime}=\left(\left\{\tau_{i j}=t_{i}^{\text {paн }}\right\} ;\left\{t_{i j}=t_{i j}^{\min }\right\}\right)$ - это целочисленная задача выпуклого кусочно-линейного программирования, которая формулируется следующим образом: в $2 r$-мерном пространстве $\tilde{R}$ найти целочисленную точку, доставляющую минимум функционала (9) при выполнении ограничений (6), (7) и (11).

Рассмотрим ограничения $\left(5^{\star *}\right)$ по ресурсам. Аргументы и параметры функций есть функция $f^{k}\left(\tau_{i j}, t_{i j}\right)$, значения которой являются целочисленными. Однако для простоты будем считать аргументы $\tau_{i j}, t_{i j}$ и параметр $k$ непрерывными, что не противоречит смыслу задачи, так как это временные величины. Параметры $N_{i j}$ дискретны по существу задачи.

На рис. 4 показана область определения функции $f^{k}\left(\tau_{i j}, t_{i j}\right)$ в плоскости $\left(\tau_{i j}, t_{i j}\right)$ (заштрихованный прямоугольник). 


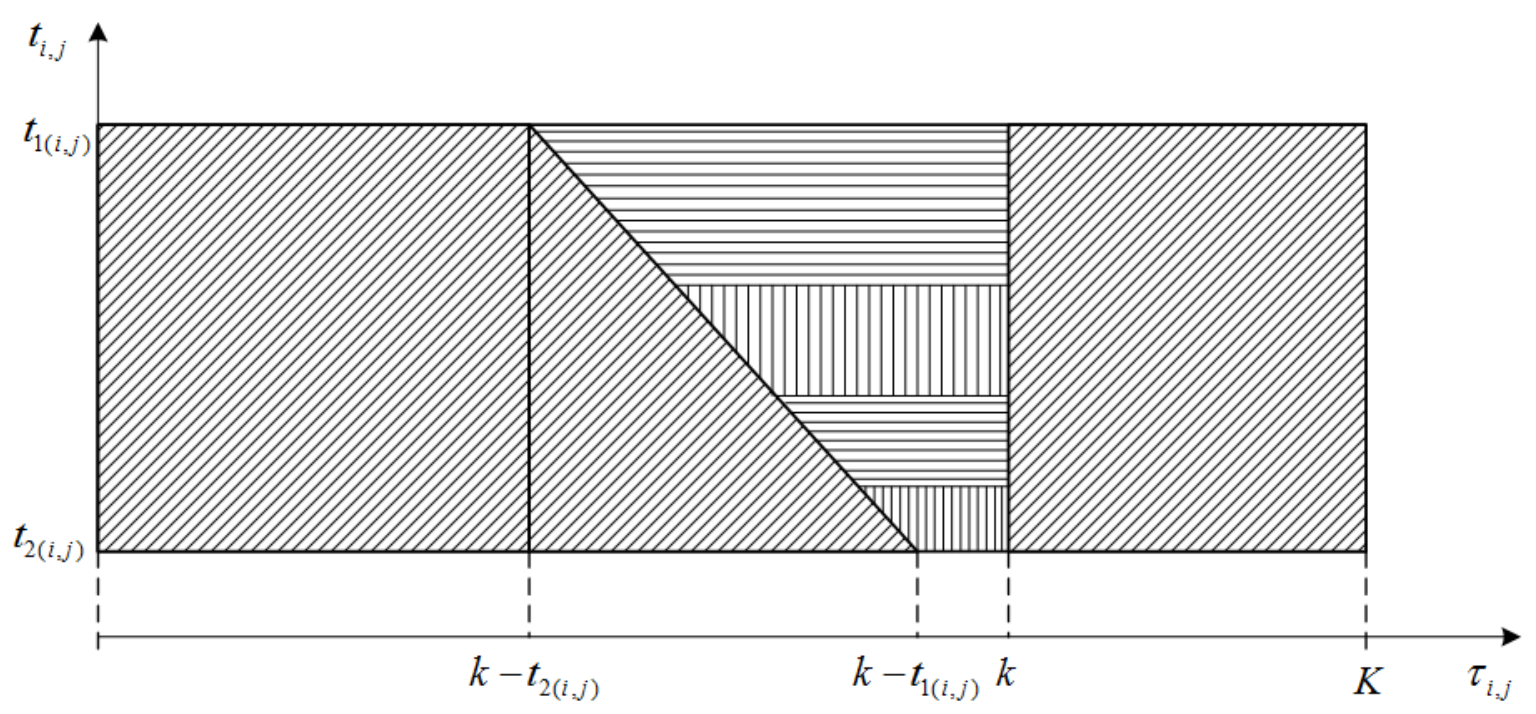

Рис. 4. Область определения функиии $f^{k}\left(\tau_{i j}, t_{i j}\right)$

На площадках с наклонной штриховкой функция $f^{k}\left(\tau_{i j}, t_{i j}\right)=0$. На площадках трапециевидной формы с прямой штриховкой она принимает постоянные положительные значения $N_{i j}\left(N_{i j}^{\min } \leq N_{i j} \leq N_{i j}^{\max }\right)$, возрастающие от площадки к площадке по мере приближения к оси $\tau_{i j}$. Следует отметить, что при погружении в подпространство пространство $\tilde{R}$ большей размерности область определения функции $f^{k}\left(\tau_{i j}, t_{i j}\right)$ будет иметь цилиндрическую форму с основанием в описанной области и образующими, перпендикулярными к плоскости $\left(\tau_{i j}, t_{i j}\right)$.

Если $L_{w}$ содержит две операции $(i, j)$ и $(\bar{i}, \bar{j})$, то область определения функции $f^{k}\left(\tau_{i, j}, t_{i, j}\right)+f^{k}\left(\tau_{\bar{i}, \bar{j}}, t_{\bar{i}, \bar{j}}\right)$ в четырехмерном пространстве $\left(\tau_{i, j}, \tau_{\bar{i}, \bar{j}} ; t_{i, j}, t_{\bar{i}, \bar{j}}\right)$ представляет собой прямоугольный параллелепипед, образовавшийся в пересечении двух цилиндрических множеств - областей определения функций $f^{k}\left(\tau_{i, j}, t_{i, j}\right)$ и $f^{k}\left(\tau_{\bar{i}, \bar{j}}, t_{\bar{i}, \bar{j}}\right)$ :

$$
\left\{\begin{array}{c}
0 \leq \tau_{i, j}, t_{i, j} \leq K, \\
0 \leq \tau_{\bar{i}, \bar{j}}, t_{\bar{i}, \bar{j}} \leq K, \\
t_{i, j}^{\min } \leq t_{i, j} \leq t_{i, j}^{\max }, \\
t_{\bar{i}, \bar{j}}^{\min } \leq t_{\bar{i}, \bar{j}} \leq t_{i, j}^{\max } .
\end{array}\right.
$$

В этом случае область, где выполняется $\left(5^{\star \star}\right)$, будет представлять один из трех типов: выпуклый прямоугольный параллелепипед; связная невыпуклая область; несвязная область, состоящая из выпуклых компонент.
Аналогичная ситуация будет при любом числе операций в $L_{w}$. Если взять теперь группу неравенств $\left(5^{\star *}\right)$ с постоянным $w$ и всеми значениями $k$ от 1 до $K$, то область решений такой подсистемы системы $\left(5^{\star *}\right)$ есть пересечение $K$ областей указанных трех типов. Это пересечение может принадлежать одному из тех же трех типов. Область решений такой подсистемы в пространстве $R$ представляет собой цилиндрическое множество с описанной областью в качестве основания. Наконец, область решений всей системы $\left(5^{\star *}\right)$ - это пересечение $n^{\prime}$ областей решений подсистем для каждого $w$, т. е. цилиндрических множеств с основаниями в $n^{\prime}$ попарно ортогональных подпространствах пространства $\tilde{R}$. Такое пересечение может быть выпуклым множеством лишь в том случае, если все $n^{\prime}$ областей решений подсистемы выпуклы. Тогда задача $\Omega^{*}$ является задачей выпуклого кусочно-линейного программирования. Во всех остальных случаях задача кусочно-линейного программирования при выпуклом функционале становится невыпуклой по ограничениям.

\section{ПРИМЕР РЕШЕНИЯ ЗАДАЧИ РАСПРЕДЕЛЕНИЯ РЕСУРСОВ}

Пусть необходимо выполнить некоторый проект $A$, заданный в виде детерминированной СМ с двумя целевыми событиями $\mu$ 
(8 и 9) и с первоначальным планом распределения ресурсов. Технология проектирования и принятый порядок выполнения операций с исходными параметрами СМ представлены на рис. 5 и табл. 1.

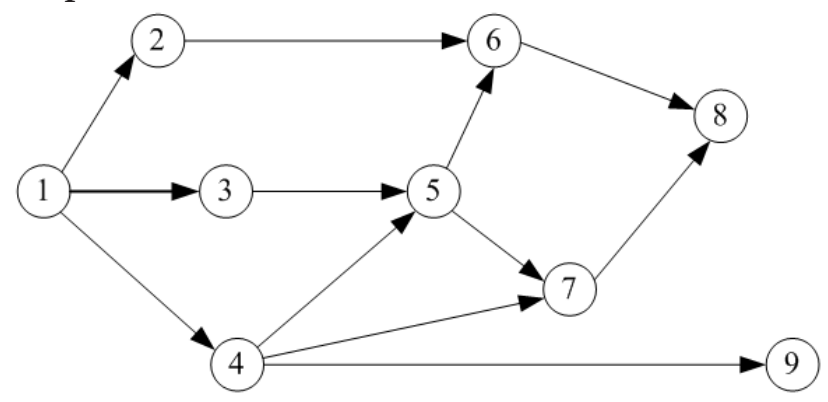

Рuc. 5. Исходная СМ

Примем, что на реализацию проекта назначены ресурсы в количестве 5 человек, $x=5$. Исполнители сгруппированы в 2 подразделения (команды) так, что в каждом подразделении все исполнители одинаковой квалификации $\left(x_{1}=2, x_{2}=3\right)$ и каждая операция СМ выполняется лишь в одном подразделении. В случае недостаточности ресурсов осуществляется их перераспределение (в одну команду). Фиктивные операции, не требующие ни времени, ни исполнителей, и операции, обозначающие процессы, требующие только времени без исполнителей формально

выполняются в подразделении без исполнителей. Условно разбив проект $A$ на операции, необходимо оптимально распределить ресурсы при заданных директивных сроках операций: $T_{8}^{\partial и р}=150$ ч и $T_{9}^{\partial и р}=140$ ч и определить новую структуру СM.

Отметим, что в частном случае задачи $\Omega$ (задача $\Sigma$ ) $N_{i j}^{\min }=N_{i j}^{\max }=N_{i j}^{\text {доn }}$ для всех операций СМ. Перепишем функционал (9) в следующем виде [3]:

$$
\begin{gathered}
F(D)= \\
=-\left(\sum_{l_{(i j)} \in T^{k p}} \sum_{p=1}^{M_{(i j)}} \frac{1}{\sum_{m \in L_{w}} \lambda_{m(i j)} \cdot x_{m p(i j)}^{*}} \cdot C_{p(i j)}-T_{\mu}^{\partial u p}\right),
\end{gathered}
$$

где $M_{(i j)}$ - число частей $l_{(i j)}$-й операции, которое обеспечит максимум ЦФ (9) в области заданных ограничений $\left(5^{*}\right),\left(6^{*}\right),\left(7^{*}\right),(11)$ соответственно, $p=1,2, \ldots, M_{(i j)}$;

$C_{(i j)}$ - доля выполненной части $l_{(i j)}$-й операции;

$x_{(i j)}$ - число исполнителей проекта $m$-го типа ( $m$-й команды), приступивших к выполнению $l_{(i j)}$-й операции (для рассматриваемого примера $m=1$ или $m=2)$;

$\lambda_{m(i j)}-$ производительность исполнителя $m$-го типа ( $m$-й команды), которая задается в частях $l_{(i j)}$-й операции. Например, для опера-

Таблица 1

\section{Исходные параметры СМ}

\begin{tabular}{|c|c|c|c|c|c|c|c|c|}
\hline$i$ & $j$ & $t_{(i j)}$, ч & $x_{1(i j)}$, чел. & $x_{2(i j)}$, чел. & $N_{1(i j)}^{\text {до }, \text { чел. }}$ & $N_{2(i j)}^{\text {доп }, \text { чел. }}$ & $\lambda_{1(i j)}$ & $\lambda_{2(i j)}$ \\
\hline 1 & 2 & 10 & 1 & 1 & 2 & 2 & 0,067 & 0,033 \\
\hline 1 & 3 & 45 & - & 1 & 3 & 2 & 0,1 & 0,0222 \\
\hline 1 & 4 & 40 & 1 & - & 2 & 2 & 0,025 & 0,05 \\
\hline 2 & 6 & 35 & 2 & 2 & 2 & 2 & 0,007 & 0,007 \\
\hline 3 & 5 & 60 & - & 1 & - & 3 & - & 0,0167 \\
\hline 4 & 5 & 20 & 3 & - & 3 & - & 0,0167 & - \\
\hline 4 & 7 & 5 & 1 & 4 & 1 & 4 & 0,04 & 0,04 \\
\hline 4 & 9 & 55 & - & 1 & 3 & 3 & 0,1 & 0,0167 \\
\hline 5 & 6 & 15 & 1 & - & 4 & 5 & 0,067 & 0,033 \\
\hline 5 & 7 & 15 & 1 & - & 2 & 2 & 0,2 & 0,04 \\
\hline 6 & 8 & 50 & - & 1 & 3 & 2 & 0,04 & 0,02 \\
\hline 7 & 8 & 50 & - & 5 & 1 & 5 & - & 0,0182 \\
\hline
\end{tabular}


Таблица 2

Полученные параметры новой СМ

\begin{tabular}{|c|c|c|c|c|c|c|c|c|}
\hline$i$ & $j$ & $t_{(i j)}$, ч & $x_{1(i j)}$, чел. & $x_{2(i j)}$, чел. & $N_{1(i j)}^{\text {доп }, \text { чел. }}$ & $N_{2(i j)}^{\text {доп }, \text { чел. }}$ & $\lambda_{1(i j)}$ & $\lambda_{2(i j)}$ \\
\hline 1 & 5 & 10 & 1 & 1 & 2 & 3 & 0,067 & 0,033 \\
\hline 1 & 3 & 40 & - & 1 & 3 & 2 & 0,1 & 0,0222 \\
\hline 1 & 2 & 40 & 1 & - & 2 & 2 & 0,025 & 0,05 \\
\hline 2 & 3 & 0 & - & - & - & - & - & - \\
\hline 2 & 6 & 20 & 3 & - & 3 & - & 0,0167 & - \\
\hline 2 & 8 & - & 4 & - & 5 & - & 0,04 & - \\
\hline 2 & 11 & 55 & - & 1 & 3 & 3 & 0,1 & 0,0167 \\
\hline 3 & 4 & 0,9 & 1 & 1 & 2 & 2 & 0,025 & 0,05 \\
\hline 4 & 6 & 60 & - & 1 & - & 3 & - & 0,0167 \\
\hline 4 & 8 & 0 & - & - & - & - & - & - \\
\hline 5 & 7 & 35 & 2 & 2 & 2 & 2 & 0,007 & 0,007 \\
\hline 6 & 7 & 15 & 1 & - & 4 & 5 & 0,067 & 0,033 \\
\hline 6 & 9 & 15 & 1 & - & 2 & 2 & 0,2 & 0,04 \\
\hline 7 & 10 & 50 & - & 1 & 2 & 2 & 0,04 & 0,02 \\
\hline 8 & 9 & - & 5 & - & 5 & - & 0,04 & - \\
\hline 9 & 10 & 50 & - & 5 & 2 & 2 & - & 0,0182 \\
\hline
\end{tabular}

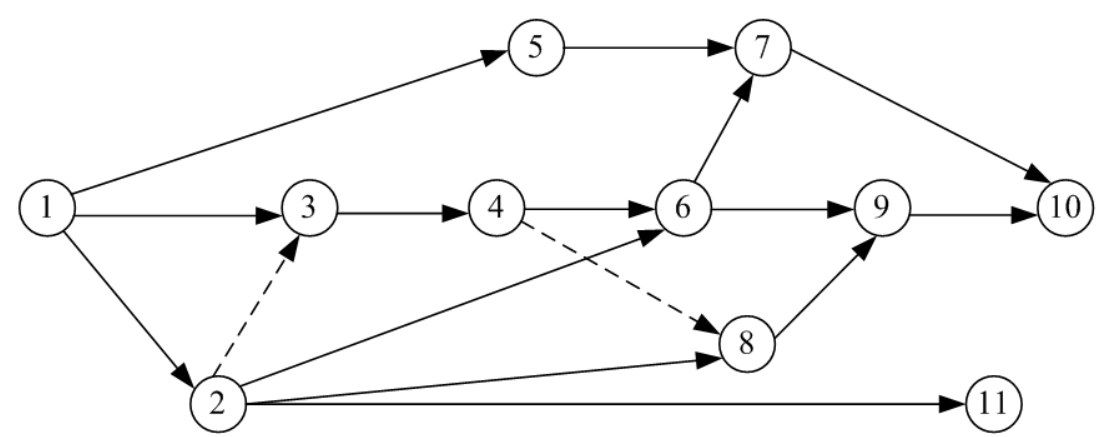

Рис. 6. Полученная СM

ции (1-3) производительность $\lambda_{1(1-3)}=0,1$. Это значит, что в единицу времени 1 исполнитель 1-го типа может выполнить 0,1 часть операции (1-3).

При распределении исполнителей по операциям СМ (рис. 5), в которой два критических пути ((1-3)-(3-5)-(5-6)-(6-8) и (1-3)(3-5)-(5-7)-(7-8)) ЦФ (12) примет значение: $F_{1}(D)=-20$ ч. Расчет параметров СМ производится в соответствии с [8] и представлен в табл. 2.

В результате решения задачи, получим новую СМ проекта $A$, представленную на рис. 6 .

Пересчитав значения ЦФ (12), получим искомое значение: $F_{2}(D)=-15,9$ ч.

\section{ОБСУЖДЕНИЕ РЕЗУЛЬТАТОВ}

В связи с тем, что СМ проектов могут состоять из большого числа операций, то для оценки предложенного подхода рассмотрим общее время вычислительного процесса на ЭВМ, которое определим по формуле:

$$
T=t_{1} n_{\text {пер }}=\frac{K_{1} K_{2} t_{(i j)} L}{\Delta t},
$$

где $t_{1}$ - время определения оптимального варианта перераспределения исполнителей в пределах двух соседних узлов;

$n_{\text {пер }}$ - число перераспределений исполнителей; 


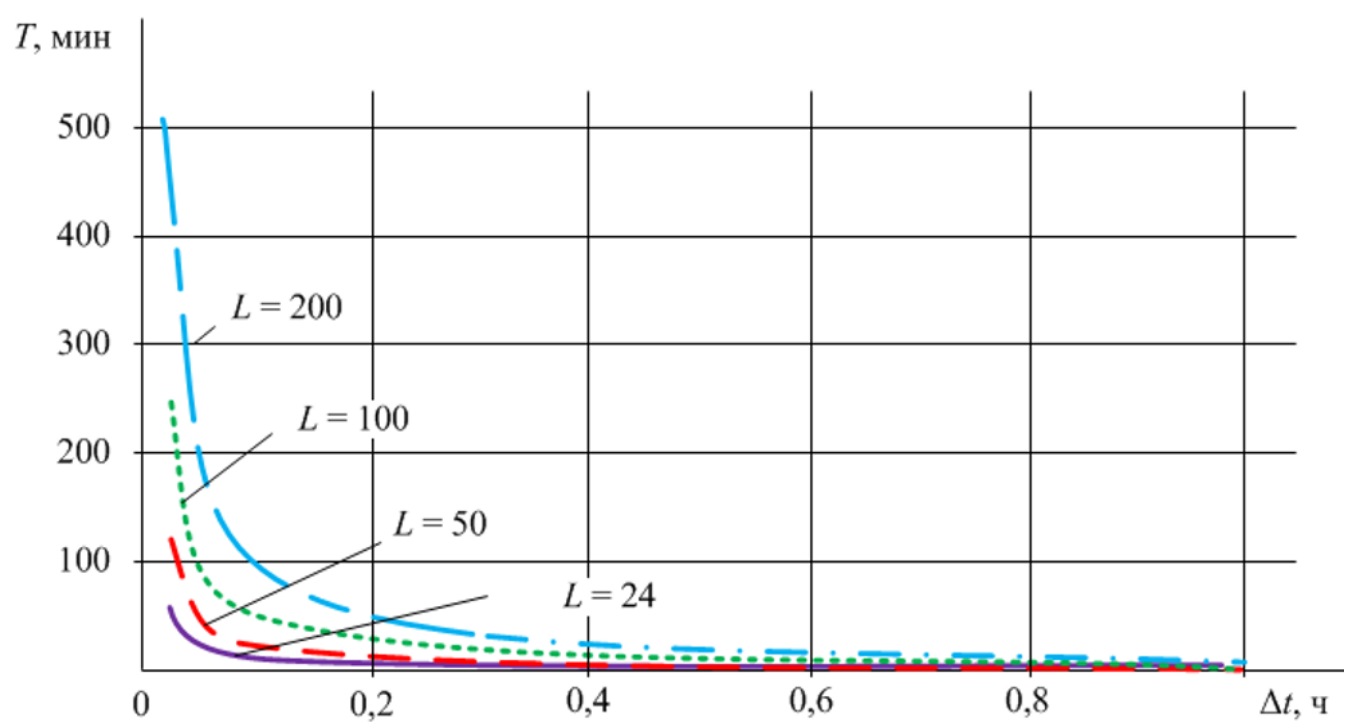

Рис. 7. Вычислительные затраты на ЭВМ в зависимости от числа операций

$K_{1}$ - коэффициент производительности ЭВМ $(0,5-0,7)$;

$K_{2}$ - коэффициент запараллеленности операций СМ $(0,2-0,4)$;

$\Delta t$ - интервал деления операций (чем меньше $\Delta t$, тем выше точность решения задачи).

На рис. 7 приведен график вычислительных затрат на ЭВМ.

Анализ графика показывает, что для СМ с небольшим числом операций интервал $\Delta t$ следует выбирать не больше 0,2 ч (единицы времени), а при большом числе операций - более 0,4 ч, поскольку при меньших значениях $\Delta t$ вычислительное время начинает возрастать в разы, тогда как снижение резервов времени будет составлять единицы процентов.

При этом, повышение эффективности при использовании предложенного подхода, может быть оценено следующим соотношением:

$$
\ni=1-\frac{F_{2}(D)}{F_{1}(D)}=1-\left(\frac{-15,9}{-20}\right)=20,5 \% .
$$

Таким образом, полученное значение ЦФ дает возможность руководителю варьировать установкой директивного срока окончания выполнения проекта и осуществлять оперативное управление.

\section{ЗАКЛЮЧЕНИЕ}

Проведенный анализ показывает, что задачи $\Omega$ и $\Sigma$ принадлежат к классу задач, для точного решения которых следует применять методы переборов. При этом использование приближенных методов в классическом смысле (для которых доказана сходимость к решению или имеются априорные оценки погрешности), не приводит к достоверным результатам. Таким образом, для решения данного класса задач следует использовать приближенные методы их решения, а именно: эвристические методы и подходы, несмотря на то, что они не имеют строгих доказательств.

\section{СПИСОК ЛИТЕРАТУРЫ}

1. Grune, L. Anetworked unconstrained nonlinear mpc scheme / J. Pannek, K. Worthmann // In: Proc.European Control Conference. - 2009. P. 91-96.

2. Masuda, N. Impact of hierarchical modular structure on ranking of individual nodes in directed networks / Y. Kawamura, H. Kori // New Journal of Physics. - 2009. - № 11. - 113002. Режим доступа: http://www.njp.org/ - (Дата обращения: 05.02.2018).

3. Коновалов, О. А. Метод оптимизации структуры детерминированной сетевой модели / О. А. Коновалов, Е. В. Коновальчук, К. А. Малыков, Ю. С. Сербулов // Вестник Воронеж. гос. ун-та. Сер. Системный анализ и информационные технологии. - 2012. - № 2. С. 111-116.

4. Коновалов, О. А. Информационные технологии управления потенциалом трудовых ресурсов: монография / О. А. Коновалов, 
Ю. С. Сербулов, О. В. Курипта; Palmirium Academic Publishing, Германия, 2016. - 220 c.

5. Афанасьев, М. Ю. Прикладные задачи исследования операций: Учеб.пособие / М. Ю. Афанасьев и др. - М.: ИНФРА-М, 2006. - 352 c.

6. Карманов, В. Г. Математическое программирование: Учеб.пособие. - 5-е изд., стереотип / В. Г. Карманов. - М. : ФИЗМАТЛИТ, 2004. -264 c.

7. Новицкий, Н. И. Сетевое планирование и управление производством: Учеб.-практ.

Коновалов Олег Анатольевич - канд. техн. наук, преподаватель ВУНЦ ВВС «Военно-воздушная академия им. проф. Н. Е. Жуковского и Ю. А. Гагарина» (г. Воронеж).

Тел.: 89081327068

E-mail: Oleg-070707@yandex.ru

Коновальчук Евгений Викторович - канд. техн. наук, доцент, начальник факультета ВУНЦ ВВС «Военно-воздушная академия им. проф. Н. Е. Жуковского и Ю. А. Гагарина» (г. Воронеж).

Тел.: 89102426919

Прохорский Руслан Александрович - канд. техн. наук, начальник 14 ЛНИ (ВНИ) НИЦ О и ИТ ВУНЦ ВВС «Военно-воздушная академия им. проф. Н.Е. Жуковского и Ю.А. Гагарина» (г. Воронеж).

Тел.: 89081302424

E-mail: Ruslan20064@rambler.ru

Винокуров Дмитрий Сергеевич - оператор научной роты ВУНЦ ВВС «Военно-воздушная академия им. проф. Н. Е. Жуковского и Ю. А. Гагарина» (г. Воронеж).

Тел.: 89055102658

E-mail: dimonv1000@mail.ru пособие / Н. И. Новицкий. - М. : Новое знание, 2004. - 159 с.

8. Свидетельство об официальной регистрации программы для ЭВМ 2014615471 РФ. Программа управления распределением ресурсов при оптимизации структуры сетевой модели / Коновалов О.А. и др. (RU); правообладатели О. А. Коновалов и др. - 2014613281; заявл. 11.04.14.; зарегистрировано в Реестре программ для ЭВМ 27.05.14.

Konovalov Oleg - candidate of technical sciences, teacher of the MESC AF "N. E. Zhukovsky and Y. A Gagarin Air Force Academy" (Voronezh).

Tel.: 89081327068

E-mail: Oleg-070707@yandex.ru

Konovalchuk Evgeniy - candidate of science, assistant professor, the chief of faculty of the MESC AF "N. E. Zhukovsky and Y. A Gagarin Air Force Academy" (Voronezh).

Tel.: 89102426919

Prokhorsky Ruslan Aleksandrovich - candidate of technical sciences, the chief of LR (MSI) SIC E IT of the MESC AF "N. E. Zhukovsky and Y. A. Gagarin Air Force Academy" (Voronezh).

Tel.: 89081302424

E-mail: Ruslan20064@rambler.ru

Vinokurov Dmitry Sergeevich - operator of a scientific company of the MESC AF "N. E. Zhukovsky and Y. A Gagarin Air Force Academy” (Voronezh).

Tel.: 89055102658

E-mail: dimonv1000@mail.ru 